\title{
Filament formation in carbon nanotube-doped lyotropic liquid crystals $\dagger$
}

\author{
Stefan Schymura ${ }^{a}$, Sarah Dölle $^{a}$, Jun Yamamoto $^{b}$ and Jan Lagerwall ${ }^{* a, c}$
}

\author{
Received Xth $X X X X X X X X X X 20 X X$, Accepted Xth $X X X X X X X X X 20 X X$ \\ First published on the web Xth $X X X X X X X X X X 200 X$ \\ DOI: 10.1039/b000000x
}

By introducing carbon nanotubes (CNTs) into a lyotropic nematic liquid crystal, strongly enhanced viscoelastic behaviour results, allowing the extraction of very thin and long filaments in which the CNTs are uniformly aligned. The filament formation requires the liquid crystallinity of the host phase and it does not take place for coarsely dispersed nanotubes or if their concentration is below a threshold value. The type of nanotube plays little role, single- as well as multiwall CNTs both triggering the filament formation, but spherical $\mathrm{C}_{60}$ fullerenes do not give rise to the phenomenon. We argue that individualized $\mathrm{CNT}_{\mathrm{N}}$ stiffen the rod-shaped micelles of the liquid crystal host and that elongational flow then increases the nematic long-range order as well as the micelle length. If the CNTs are present at a sufficient concentration to connect into continuous linear chains of arbitrary extension, the micelle stiffening is ensured regardless of length, taking the system into a positive feedback loop between increasing orientational order and diverging micelle length. It is this percolation-like transition to aligned and quasi-infinite micelles stabilized by chains of nanotubes that makes the filament formation possible.

\section{Introduction}

Whether for research or for applications, carbon nanotubes (CNTs $)^{1}$ are generally processed as liquid dispersions. Due to the extreme nanotube anisometry and consequent anisotropy in the physical properties it is often important to control the CNT orientation, a requirement that is not trivial to fulfil. A convenient solution taking the liquid-dispersed state as starting point is to use an ordered fluid, a liquid crystal, for the dispersion. CNTs that are well dispersed in a liquid crystal (LC) spontaneously align along the director $\mathbf{n}$, the preferred orientation of the host phase ${ }^{2-6}$. We recently demonstrated that an efficient approach is to incorporate CNTs dispersed with the anionic surfactant sodium dodecylbenzenesulfonate (SDBS) in an aqueous lyotropic LC phase formed by the cationic surfactant hexadecyltrimethylammonium bromide $(\mathrm{CTAB})^{7,8}$. With this combination liquid crystalline dispersions of single- as well as multiwall nanotubes can be prepared at sufficiently high concentration, with large-scale uniform alignment, that the composite fluid acts as a linear polariser, cf. Fig. 1a. The effect reflects the fact that CNTs absorb light polarised along

$\dagger$ Electronic Supplementary Information (ESI) available: Movie of filamen drawing from a CNT-loaded LC. See DOI: 10.1039/b000000x/

${ }^{a}$ Martin-Luther-University Halle-Wittenberg, Institute of Chemistry - Physical Chemistry, von-Danckelmann-Platz 4, 06120 Halle, Germany. ${ }^{b}$ Division of Physics and Astronomy, Graduate School of Science, Kyoto University, Kitashirakawa-Oiwakecho, Sakyo-ku, Kyoto 606-8502, Japan. ${ }^{c}$ Seoul National University, Graduate School of Convergence Science and Technology, Suwon-si, Gyeonggi-do, Korea 443-270. Fax: +82 (0)31 888 9155; Tel: +82 (0)31 888 9165; E-mail: jan.lagerwall@ lcsoftmatter.com the tube axis but not perpendicularly polarised light, hence the absorption by the fluid with uniformly aligned nanotubes becomes polarisation dependent. ${ }^{7,8}$

Interestingly, these suspensions also display a strong tendency to form thin and very long filaments when subjected to elongational flow (Fig. 1b, ESI 1). This filament formation is fascinating from a basic physicochemical point of view, but it may also be attractive for CNT fibre production or for transferring LC-aligned CNTs to target substrates in a controlled manner since the contained tubes are very well aligned along the filament ${ }^{7,8}$. The drawing can be done very fast, such that a filament with length on the order of $1 \mathrm{~m}$ can be extracted from the bulk sample in about a second. This can be compared with a previous report of CNT fiber drawing directly from isotropic nanotube suspensions, allowing a drawing rate of only $0.1 \mathrm{~mm} /$ minute $^{9}$ (Other ways of producing CNT fibres, involving quite different mechanisms, also allow fast production rates, see e.g. references ${ }^{10,11}$.) The extended filament is stable enough to be deposited in a selected direction, if desired with curvature, on any given substrate. If the process can be fully understood and made compatible with an industrially viable production technique (e.g. spinning, microfluidics or automated fluid dispensers) this could be a new opening towards CNT applications.

The purpose of the present work is to provide a first step towards such an understanding. To this end we have systematically investigated the filament formation process, varying nanotube type, concentration as well as the nature of the host phase. We find that any type of carbon nanotube induces the 


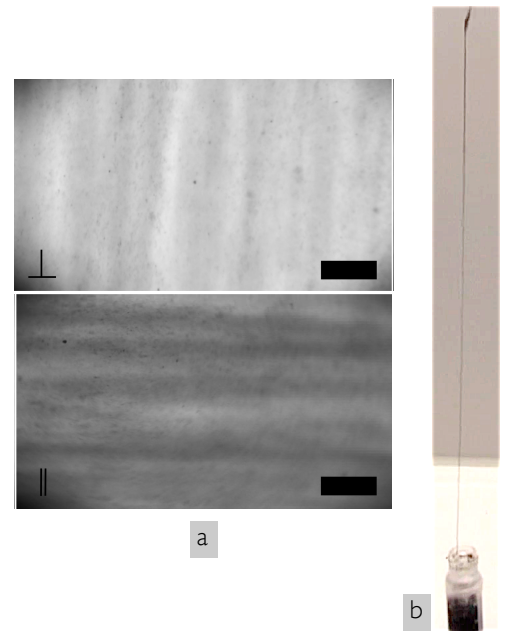

Fig. 1 (a) Polarising effect of a shear aligned CNT/LC phase with $28 \mathrm{wt} \%$ CTAB and $2.5 \mathrm{mg} / \mathrm{mL}$ SDBS-stabilised SWCNTs. The sample is illuminated by unpolarised light but viewed through a polariser that is perpendicular (top) and parallel to the director (bottom), respectively. Scale bar $=100 \mu \mathrm{m}$. (b) Thin filament drawn out of a bulk sample of the lyotropic CNT/LC composite.

filament formation in nematic phases but not in isotropic hosts. No filaments develop if the nanotubes are replaced by spherical $\mathrm{C}_{60}$ fullerenes. Moreover, the CNTs need to be very well dispersed and present above a threshold concentration. We can explain the phenomenon as a result of a combination of events, CNT-induced micelle stiffening and nanotube chain formation being crucial components together with the elongational flow and the orientational order of the nematic host phase.

\section{Experimental}

Two types of single-wall carbon nanotube (SWCNT) and two different samples of multiwall carbon nanotube (MWCNT) were used in this work. The majority of experiments were conducted with SWCNTs of Hipco type (Unidym/CNI, USA, $<15 \%$ ash content) but also CoMoCat SWCNTs (SWeNT, USA, 1-14 wt-\% catalyst residues) were investigated. Both SWCNT types have a diameter of about $1 \mathrm{~nm}$ and length in the range hundreds of nanometers to a few microns. The MWCNT samples (SES Research, USA, $<0.2 \%$ ash content) both have lengths in the range $5-15 \mu \mathrm{m}$, while their diameter distributions are in the ranges $10-30 \mathrm{~nm}$ and $60-100 \mathrm{~nm}$, respectively. For reference, experiments were also done on spherical $\mathrm{C}_{60}$ fullerenes (SES Research, USA, 99.9\% nominal purity) added to the host phase instead of nanotubes.

The CNTs were first dispersed at a concentration of $2.5 \mathrm{mg} / \mathrm{mL}$ in aqueous SDBS solution, with a ratio of surfactant to CNTs of 10:1 by weight, using a Dr. Hielscher
UIS205L sonotrode working continuously at full power. The $10 \mathrm{~mL}$ sample was water cooled during the process in order to avoid excessive temperature increase and the sonication was pursued until no aggregates could be perceived by optical microscopy, typically about three hours for this relatively large volume. Small subsamples of this mother dispersion were used as is or diluted with SDBS solution for obtaining samples with different nanotube concentrations, and to each subsample was added a selected amount of CTAB. The final CTAB concentration was tuned to the range 15 28 wt.- $\%$, where concentrations above 23 wt.- $\%$ lead to the development of a nematic liquid crystalline phase ${ }^{12}$. Even higher CTAB concentrations, where a hexagonal phase would develop, were not investigated. Immediately after adding the dispersion of SDBS-stabilised CNTs to the dry CTAB the mixture was stirred using a magnetic stirrer for at least one hour at somewhat elevated temperature $\left(40^{\circ} \mathrm{C}\right)$ until homogenous by eye. This coarse homogenisation was followed by a second sonication, this time in a Bandelin Sonorex TK52 sonication bath for about three hours, resulting in homogeneous mixtures. The reference samples loaded with $\mathrm{C}_{60}$ fullerenes were prepared following the same protocol.

Filaments were drawn using a metallic spatula or a pasteur pipette emerged into and then retracted from the CNT/LC composite fluids. Rheological data were recorded using an Anton Paar Physica MCR-301 Rheometer. For microscopy investigation the fluid was filled by light vacuum suction into an optically flat capillary, the shear flow during filling ensuring an orientation of $\mathbf{n}$ along the capillary.

\section{Results}

We first point out that without nanotubes the LC phase does not show any tendency whatsoever to form filaments. Neither from the pristine $\mathrm{LC}$ nor from the phase loaded with $\mathrm{C}_{60}$ fullerenes can filaments be drawn. The CNTs are thus a prerequisite for the phenomenon to occur and their concentration must be above a certain threshold $c_{T}$. For Hipco SWCNTs we found this to be in the range $0.5-1 \mathrm{mg} / \mathrm{mL}$. In the lower part of this range unstable filaments can be drawn with a maximum length on the order of centimetres. At a concentration of $1 \mathrm{mg} / \mathrm{mL}$ or higher it becomes easy to draw very long and stable filaments. While CNTs are thus necessary for the phenomenon the type of carbon nanotube is not important. With all four CNT samples investigated in this study, SW- as well as MWCNTs, filaments could be drawn from a nematic phase loaded with the tubes, although the threshold value may be somewhat different (it was quantitatively established only for the case of Hipco SWCNTs).

In contrast to CNT type the quality of the dispersion of the nanotubes in the LC phase is important-critically so-for filament formation. In fact, the possibility to draw long filaments 


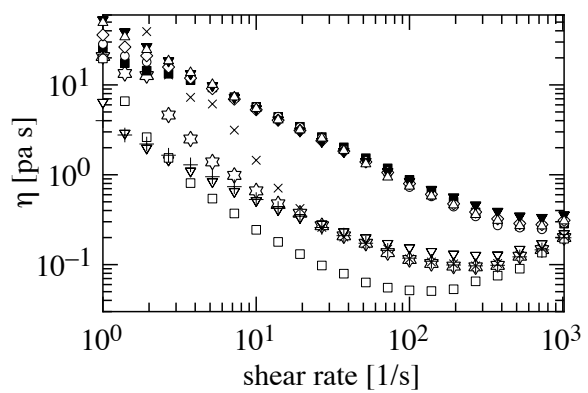

Fig. 2 Shear viscosity $\eta$ of HiPCO CNT/LC phases as a function of shear rate and nanotube concentration. The CTAB concentration is in all cases $28 \mathrm{wt} \%$.

together with verification of the polarising effect demonstrated in Fig. 1a have become our standard two tests for sufficient nanotube dispersion quality. The polarising effect requires that the nanotubes are aligned by the LC and this does not happen in case of poorly dispersed tubes gathered in optically visible aggregates, which thus absorb light irrespective of the light polarisation. The reason why no filaments can be drawn in case of insufficient dispersion quality is less obvious but after the analysis of the process to be carried out in the following we can understand also this observation.

The liquid crystalline nature of the host fluid is also a requirement for the formation of filaments. With the isotropic low-CTAB-concentration samples filaments could generally not be drawn. Only if the bulk surfactant concentration was just below the threshold for steady-state nematic ordering was it possible to draw filaments from a phase that appears macroscopically isotropic at rest, but the filaments were very likely liquid crystalline also in this case. It is well known that CTAB solutions of this kind, at concentrations just below the onset of liquid crystallinity, exhibit a shear-induced transition to a nematic phase, ${ }^{13}$ hence the flow during filament formation may well have sufficed to take the system to an LC state. Additionally, as will be discussed below, the presence of the CNTs promotes orientational order under elongational flow, thus further supporting the formation of a nematic phase in the drawing process. Finally, the greatly increased surface area during filament formation leads to enhanced water evaporation, thus possibly dynamically taking the surfactant concentration above the threshold to the static nematic phase.

The nematic CTAB phase loaded with enough CNTs to allow filament drawing still flows relatively easily, deforming under the influence of gravity after a slight time delay if the vial containing it is turned over. A more careful rheological investigation reveals that the nanotubes have a distinct impact on the shear viscosity $\eta$, with a nontrivial concentration dependance as shown in Fig. 2 for the case of Hipco nanotubes. The

pristine CTAB phase (star symbols) exhibits a shear thinning cross-over from a high- to a low- $\eta$ regime at a critical shear rate $\dot{\gamma}_{c}$ in the range of $1 s^{-1}$. This phenomenon, which has been described previously, ${ }^{14}$ can be understood as the result of the macroscopic-scale uniform alignment of $\mathbf{n}$ and consequent reduction in micelle entanglement, possibly connected to breakage of micelles, at shear rates above $\dot{\gamma}_{c}$. At the very small SWCNT concentration of $0.05 \mathrm{mg} / \mathrm{mL}$, far below $c_{T}$, the cross-over behaviour disappears and the data now generally fall in the low- $\eta$ regime. A slightly increased viscosity compared to the pristine CTAB phase is observed only at shear rates on the order of $100 \mathrm{~s}^{-1}$. On doubling the CNT concentration $\eta$ is now distinctly lower than in the pristine phase at all but the highest shear rates studied. On further increase of the nanotube content the viscosity at $0.25 \mathrm{mg} / \mathrm{mL}$ again rises but there is still no cross-over behavior, the data generally being very similar to those at $0.05 \mathrm{mg} / \mathrm{mL}$ loading.

At $0.5 \mathrm{mg} / \mathrm{mL}$, the degree of loading where the first signs of filament formation can be found, a cross-over behavior can again be identified, now with a critical shear rate on the order of $10 s^{-1}$ separating a high- $\eta$ static system from a system that is less viscous under shear. From $c \geq 1 \mathrm{mg} / \mathrm{mL}$ the rheological properties of the composite are more uniform in character without any clear cross-over tendency, but now the data fall in a high- $\eta$ regime at all rates, in fact slightly more viscous than the sub- $\dot{\gamma}_{c}$ regime of the pristine sample. Upon further increase of the CNT concentration very little changes in the spectrum. Only at very low rates $\left(10-30 s^{-1}\right)$ can a concentration dependence still be identified, $\eta$ there generally increasing with greater CNT loading. Moreover, whereas a minimum at shear rates around $100 \mathrm{~s}^{-1}$ was found at all lower concentrations there is a monotonic decrease in viscosity up to about $1000 \mathrm{~s}^{-1}$ for $c \geq 1 \mathrm{mg} / \mathrm{mL}$. Obviously, the threshold seen at $c_{T}$ for filament drawing corresponds to a transition to a new type of behaviour in terms of the rheological properties.

As mentioned previously the type of CNT has little impact on the possibility to draw filaments from the CNT-LC composite. The same independence is seen in the rheological properties, the viscosity $v s$. shear rate curves at $2.5 \mathrm{mg} / \mathrm{mL} \mathrm{CNT}$ concentration being essentially identical for all investigated nanotube types, cf. Fig. 3 .

\section{Discussion}

The observations described above suggest that elongational flow applied to a lyotropic nematic phase containing a sufficient amount of well dispersed nanotubes induces some kind of extended linear structure formation that supports the filament as it is extended. We can find an explanation to this phenomenon in theoretical treatments of linear growth of rodlike micelles treated as a case of supramolecular polymerization. ${ }^{15,16}$ In the case of stiff and long rod micelles elongational 


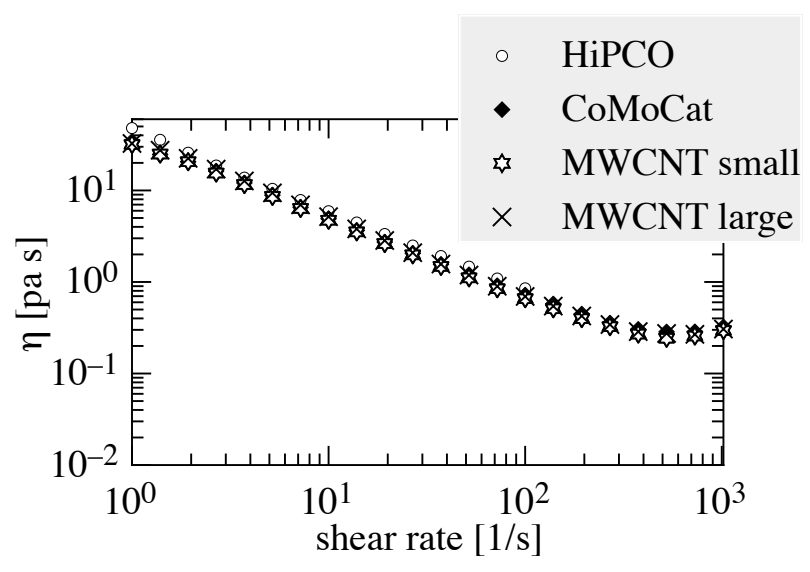

Fig. 3 Shear viscosity $\eta$ of CNT/LC phases as a function of shear rate and CNT type. The CTAB concentration is in all cases $28 \mathrm{wt} \%$, the CNT concentration $2.5 \mathrm{mg} / \mathrm{mL}$.

flow promotes orientational order, which in turn promotes further linear growth of the micelles, which further reduces the rotational freedom and thus the orientational order. A positive feedback loop arises that under the right conditions leads to a divergence of the rod length and a very high degree of orientational order. ${ }^{15}$

To apply these theoretical findings to our case we identify the monomer with a rodlike micelle of CTAB, growing in length ('polymerising') by fusing with adjacent micelles, as in the case considered by Turner and Cates ${ }^{16}$. The crucial role of the nanotubes is to give the monomer micelles the required exceptional length and stiffness, a situation which has been confirmed through cryo electron microscopy investigations by Regev and co-workers for CTAB micelles in the vicinity of carbon nanotubes. ${ }^{17,18}$. The role of the nematic host phase is to provide a quadrupolar ordering field that was recognized by van der Schoot as a key factor in the divergence of the effective chain length. ${ }^{15}$

As an alternative mechanism one could in principle contemplate that the filament is supported directly by linearly aggregating CNTs, without the need for fusing of surrounding CTAB micelles. In surfactant stabilized CNT dispersions depletion attraction may be quite strong ${ }^{19,20}$, as surplus surfactant micelles act as non-interacting particle species that are depleted from the space between the dispersed CNTs. This builds up an osmotic pressure that promotes aggregation of the nanotubes since aggregation would reduce the excluded volume for the micelles. ${ }^{21}$ In our special case of an anisotropic environment for the nanotubes, provided by the liquid crystalline host phase, also the depletion forces become anisotropic, being stronger along $\mathbf{n}$ than in the perpendicular directions. ${ }^{22,23}$ The tubes should thus be attracted to each other end-to-end and could effectively link up into a linear chain growing along $\mathbf{n}$. However, this scenario does not require the particles to be rod-shaped; only the host phase needs to be anisotropic. ${ }^{22,23}$ One should thus expect the same phenomenon to take place for a $\mathrm{C}_{60}$ fullerene-loaded LC phase. In contrast, we could in no case draw filaments from our reference samples of this type, as mentioned above, hence we can rule out anisotropic depletion attraction as the dominant factor behind the filament formation. We will see in the following, however, that it most likely plays a secondary role in the process.

The shear viscosity studies of bulk samples with varying amounts of nanotubes indicate distinctly different viscoelastic behaviour at concentrations below and above $c_{T}$, the minimum concentration for filament formation. The identification of such a threshold in both series of experiments suggests that the filament formation can be considered as a kind of percolation transition, i.e. the establishment of macroscopic connectivity when the nanotube concentration reaches a certain minimum value. In fact, Turner and Cates ${ }^{16}$ referred to the case of diverging micelle length as gelation, a phenomenon that can be described in general terms as an example of percolation. The percolation threshold $c_{T}$ is in our case in the range $0.5-1 \mathrm{mg} / \mathrm{mL}$, translating to a CNT volume fraction threshold in the range 0.0004 to 0.0007 (calculated with an average CNT density of $1.35 \mathrm{~g} / \mathrm{mL}$ ).

The percolation phenomenon in our case is quite different from that which is typically considered in studies of percolation in isotropic CNT suspensions. The addition of nanotubes does not lead to a general stiffening of all micelles in the host phase, only of those in the direct vicinity of a tube. At low CNT concentrations a certain degree of local micelle stiffening thus takes place, sufficient to counteract large-scale micelle entanglements and thereby reduce the viscosity at low shear rates. But as the micelles start growing in length due to the combination of increased stiffness, orientational order, and the initiating elongational flow, they do so by fusing with micelles not containing any nanotubes. The general rigidity of the micelle then decreases again, breaking the feedback loop that could otherwise have led to a divergence in micelle length. Only if the nanotubes are present at such a high concentration that they can link into an essentially quasi-infinite linear chain, i.e. sufficient to ensure macroscopic connectivity, can they provide the required micelle stiffening throughout the length divergence process. As described above such chain formation is expected as a result of the anisotropic depletion attraction forces. The minimum concentration for the chains to reach arbitrary lengths defines the percolation threshold for the filament formation.

Isotropic SWCNT suspensions have previously been shown to form physical gels for concentrations above a rigidity percolation threshold of around 0.0026 of CNT volume fraction $^{24}$. In our case the threshold is an order of magnitude 
lower, also compared to reports on electrical CNT percolation $^{25}$. This may at first sight seem surprising since we here have orientationally ordered nanotubes (Fig. 1a) while theory generally predicts that the percolation threshold is lowest for complete orientational disorder. ${ }^{19,26}$. The percolation threshold can however be strongly influenced by attractive interactions between the nanotubes. It has for instance been demonstrated that the 'stickiness' that arises from depletion attraction in high-concentration surfactant solutions strongly reduces the threshold. ${ }^{20}$ The stickiness in our case is even stronger, as it in addition is related to the micelle fusion/orientational order feedback loop during the elongational flow, and as the depletion attraction forces are anisotropic with a maximum along $\mathbf{n}$. The low value of $c_{T}$ thus becomes understandable.

As we start moving the spatula or pipette out of the bulk sample it is initially capillary forces that stabilize the protrusion, which at first is thick and not very long. But the flow aligns the director macroscopically along the drawing direction, thereby aligning also the CNTs in this direction, their degree of order in fact very likely being greater than that of the nematic matrix. ${ }^{15,16}$ The macroscopically uniform orientational order promotes linear aggregation of CNTs into longer entities which stabilize the fusing micelles, and as a result the degree of orientational order in turn increases, leading to the positive feedback loop. A chain of nanotubes and micelles of ever increasing length builds up that stabilize the filament against the collapse promoted by surface tension. This makes the extreme filament extension to lengths on the order of meters possible, provided that the CNT concentration is sufficient to allow quasi-infinite connectivity (percolation) along $\mathbf{n}$.

As discussed above, the chain of CNTs can be regarded as a long extended polymer chain, its flexibility being greater than that of a single CNT due to the loose joints between the chain links. Filament formation can be induced by adding long-chain polymers to isotropic solvents. ${ }^{27}$ In our case the combination of anisotropic solvent, anisometric and stiff nanotube additives, and elongational flow is required for the supramolecular polymerisation/chain formation to take place, but from then on the phenomena have some similarities.

Switching the type of SWCNT or even going to MWCNTs with up to 100-fold greater diameter obviously does not greatly influence the situation. While this insensitivity to CNT type may at first seem somewhat surprising, it is in fact reasonable since the anisometry even of MWCNTs is exceptional. They are also aligned by the liquid crystal along the director $^{8}$ and they can thus also be expected to have the required stiffening effect on the micelles and form chains parallel to n. In contrast, spherical $\mathrm{C}_{60}$ fullerenes will not stiffen the micelles, even if they are expected to build linear chains due to anisotropic depletion attraction, ${ }^{22,23}$ hence they cannot induce the filament formation.

If the CNTs are too poorly dispersed to be aligned in the LC no filaments are formed regardless of nanotube type. We can now easily understand this observation. Large random CNT aggregates are not stiff rods and will thus not promote the positive feedback loop between orientational order and chain formation. In addition, such aggregates disturb the director field $^{28}$ with reduced effective orientational order as consequence. Moreover, if the CNTs are large-scale aggregated the number density is much lower and the tubes are not free to reconfigure in a thinning filament to form a quasi-infinite chain.

Turning finally to the bulk shear viscosity measurements the observation that low concentrations of CNTs actually decreases $\eta$ is quite remarkable. As suggested above we propose that this is due to the ordering effect of the CNTs on the surrounding CTAB micelles. ${ }^{17,18}$ They are generally longer and extend linearly over greater distances than in the corresponding nanotube-free CTAB solution. With extended average micelle length the critical shear rate for macroscopic alignment and thus for cross-over to a less viscous regime is reduced, just like in pristine CTAB solutions upon increasing the surfactant concentration ${ }^{14}$. In our case the resulting $\dot{\gamma}_{c}$ is apparently lower than the shear rates considered in Fig. 2 already at $0.05 \mathrm{mg} / \mathrm{mL}$ CNTs. Moreover, the increased stiffness of micelles supported by CNTs should reduce the entanglement between micelles. This explains how the mixture with $0.1 \mathrm{mg} / \mathrm{mL}$ added CNTs can have viscosity values below those of the pristine CTAB solution.

Upon approaching $c_{T}$ the observed transition to a new high$\eta$ regime can be understood as the result of the linear CNT percolation and micelle length divergence that also stabilize the filaments when the nanotube concentration exceeds this threshold. Although the positive feedback loop between orientational order and linear growth should not be as pronounced in shear flow as in elongational flow, a similar but weaker effect is still expected. ${ }^{16}$ At the borderline concentration of $0.5 \mathrm{mg} / \mathrm{mL}$ macroscopic connectivity has obviously not quite been reached, as evidenced by very small maximum filament length and a viscosity that crosses over to the low- $\eta$ regime at shear rates higher than $\sim 10 s^{-1}$. But at higher nanotube concentrations the chains persist, raising the viscosity at all frequencies investigated.

\section{Conclusions and Outlook}

We showed that filament formation in CNT/LC composites is possible regardless of CNT-type, provided good dispersion quality and a CNT concentration above a threshold value $c_{T}$. As explanation we propose that the filaments are stabilized by linear chains of nanotubes and micelles of diverging length, dynamically built up in a process that can be seen as supramolecular linear polymerization or 1D percolation, made possible by the presence of long stiff rods in a uniformly oriented nematic host phase subjected to elongational flow. 
We have in this paper focused on the physicochemical processes behind the filament formation and the influence of CNT type and concentration as well as the properties of the host phase. A more technically oriented fine-tuning of the process is a logical next step for improving control and reproducibility and reaching even longer and thinner filaments. It may for instance be beneficial to use tailor-designed tips for the extraction, such as tapered tungsten wires. ${ }^{9}$ For achieving arbitrarily long filaments one could pump the CNT/LC phase to the drawing well using a microfluidic set-up, ${ }^{8}$ allowing continuous replenishment of the source fluid.

\section{Acknowledgments}

This work was supported by the "Excellenzcluster nanostrukturierte Materialien" of the Land Sachsen-Anhalt and the BIEP (Bilateral International Exchange program) of the Grant-in-Aid for the Global COE Program "The Next Generation of Physics, Spun from Universality and Emergence" from the Ministry of Education, Culture, Sports, Science and Technology (MEXT) of Japan.We thank Paul van der Schoot for highly helpful discussions and suggestions.

\section{References}

1 R. Saito, M. S. Dresselhaus and G. Dresselhaus, Physical properties of carbon nanotubes, Imperial College Press, UK, 1998.

2 G. Scalia, ChemPhysChem, 2010, 11, 333-340.

3 J. P. F. Lagerwall and G. Scalia, J. Mater. Chem., 2008, 18, 2890-2898.

4 M. Rahman and W. Lee, J. Phys. D-Appl. Phys., 2009, 42, 063001.

5 C. Zakri, Liq. Cryst. Today, 2007, 16, $1-11$.

6 J. P. F. Lagerwall, G. Scalia, M. Haluska, U. Dettlaff-Weglikowska, S. Roth and F. Giesselmann, Phys. Stat. Sol. (b), 2006, 243, 3046-3049.

7 G. Scalia, C. von Bühler, C. Hägele, S. Roth, F. Giesselmann and J. P. F. Lagerwall, Soft Matter, 2008, 4, 570-576.

8 S. Schymura, E. Enz, S. Roth, G. Scalia and J. P. F. Lagerwall, Synth. Met., 2009, 159, 2177-2179.

9 E. Y. Jang, T. J. Kang, H. Im, S. J. Baek, S. Kim, D. H. Jeong, Y. W. Park and Y. H. Kim, Adv. Mater, 2009, 21, 4357-4361.

10 K. Koziol, J. Vilatela, A. Moisala, M. Motta, P. Cunniff, M. Sennett and A. H. Windle, Science, 2007, 318, 1892-1895.

11 B. Vigolo, a. Penicaud, C. Coulon, C. Sauder, R. Pailler, C. Journet, P. Bernier and P. Poulin, Science, 2000, 290, 1331-1334.

12 G. Hertel and H. Hoffmann, Progr. Colloid Polym. Sci., 1988, 76, $123-$ 131.

13 E. Cappelaere, R. Cressely and J. Decruppe, Colloids and Surfaces A, 1995, 104, 353-374.

14 L. Coppola, R. Gianferri, I. Nicotera, C. Oliviero and G. Ranieri, Phys. Chem. Chem. Phys., 2004, 6, 2364-2372.

15 P. P. A. M. van der Schoot, Supramolecular Polymers, Taylor \& Francis, Boca Raton, USA, 2005, pp. 77-106.

16 M. Turner and M. Cates, J Phys-condens Mat, 1992, 4, 3719-3741.

17 E. Nativ-Roth, R. Yerushalmi-Rozen and O. Regev, Small, 2008, 4, 14591467.

18 E. Nativ-Roth, O. Regev and R. Yerushalmi-Rozen, Chem. Commun., 2008, 2037-2039.

19 C. Zakri and P. Poulin, J. Mater. Chem., 2006, 16, 4095-4098.
20 B. Vigolo, C. Coulon, M. Maugey, C. Zakri and P. Poulin, Science, 2005, 309, 920-923.

21 D. Marenduzzo, K. Finan and P. R. Cook, J. Cell Biol., 2006, 175, 681686.

22 P. van der Schoot, J. Chem. Phys., 2002, 117, 3537-3540.

23 P. van der Schoot, J. Chem. Phys., 2000, 112, 9132-9138.

24 L. Hough, M. Islam, P. Janmey and A. Yodh, Phys. Rev. Lett., 2004, 93, 168102.

25 F. Du, J. Fischer and K. Winey, Phys. Rev. B, 2005, 72, 121404.

26 A. Kyrylyuk and P. van, der Schoot, Proc. Natl. Acad. Sci. USA, 2008, 105, 8221-8226.

27 Y. Amarouchene, D. Bonn, J. Meunier and H. Kellay, Phys. Rev. Lett., 2001, 86, 3558-3561.

28 J. P. F. Lagerwall, G. Scalia, M. Haluska, U. Dettlaff-Weglikowska, S. Roth and F. Giesselmann, Adv. Mater., 2007, 19, 359-364. 